\title{
Causal associations of autoimmune thyroiditis and papillary thyroid carcinoma: mRNA expression of selected nuclear receptors and other molecular targets
}

\author{
DANA MACEJOVA ${ }^{1}$, JAN PODOBA ${ }^{2}$, LUCIA TOPOROVA ${ }^{1}$, MARIANNA GRIGEROVA ${ }^{2}$, \\ KAROL KAJO $^{3}$, KATARINA MACHALEKOVA ${ }^{3}$ and JULIUS BRTKO ${ }^{1}$
}

\begin{abstract}
${ }^{1}$ Department of Endocrine Regulations and Psychopharmacology, Institute of Experimental Endocrinology, Biomedical Research Center, Slovak Academy of Sciences, 84505 Bratislava; Departments of ${ }^{2}$ Endocrinology and ${ }^{3}$ Pathology, Slovak Medical University and St. Elisabeth Cancer Institute, 81250 Bratislava, Slovak Republic
\end{abstract}

Received December 7, 2018; Accepted May 29, 2019

DOI: $10.3892 / \mathrm{ol} .2019 .10770$

\begin{abstract}
Potential causal associations of autoimmune thyroiditis (AIT) and papillary thyroid carcinoma (PTC) have been studied previously. The mRNA expression patterns of thyroid hormone receptors (TR), retinoid receptors (RAR), rexinoid receptors (RXR), dihydroxyvitamin $\mathrm{D}_{3}$ receptors (VDR), and progesterone receptors (PR) in PTC tissue of patients without autoimmune thyroiditis (PTC/AIT-) and in PTC tissue of patients with coexisting AIT (PTC/AIT+) have been investigated in order to judge whether the observed changes may take part in the promotion and progression of thyroid cancer. Tumours with or without AIT were classified histologically and the semiquantitative PCR was performed. The results revealed that there was decreased expression of $\mathrm{TR} \alpha, \mathrm{TR} \beta \alpha, \mathrm{RAR} \alpha$ and PR mRNA in PTC/AIT+ tumours when compared with PTC/AIT- tumours. Decreased expression of RAR $\alpha$ in PTC/AIT+ was detected when compared with PTC/AIT- patients. A similar effect of AIT was observed with a decrease in RAR $\gamma$ expression in PTC/AIT+ patients. On the other hand, there was an increased expression of VDR in thyroid tumours (PTC/AIT+) when compared with PTC/AIT-. PR mRNA was decreased in the thyroid tumours of PTC/AIT+
\end{abstract}

Correspondence to: Dr Dana Macejova, Department of Endocrine Regulations and Psychopharmacology, Institute of Experimental Endocrinology, Biomedical Research Center, Slovak Academy of Sciences, Dubravska cesta 9, 84505 Bratislava, Slovak Republic E-mail: ueenmace@savba.sk

Abbreviations: AIT, autoimmune thyroiditis; COX-2, cyclooxygenase-2; DIO1, type I iodothyronine 5'-deiodinase; IGF-1, insulin-like growth factor-I; PR, progesterone receptor; PTC, papillary thyroid carcinoma; RAR, retinoid receptor; RXR, rexinoid receptor; TR, thyroid hormone receptor; VDR, dihydroxyvitamin $\mathrm{D}_{3}$ receptor

Key words: papillary thyroid carcinoma, autoimmune thyroiditis, causality, nuclear receptors, reverse transcription-semiquantitative PCR patients when compared with PTC/AIT- patients. In addition, there was an increased expression of MKi67 and complement $\mathrm{C} 3$ in PTC of PTC/AIT+ when compared with PTC/AIT-. In the PTC/AIT+ group, a decreased level of IGF-1 mRNA was found when compared with the PTC/AIT- group. According to the significant differences of the studied markers in PTC/AIT+ compared with PTC/AIT-, it was indicated that AIT may be a predisposing factor for the development of PTC.

\section{Introduction}

Several lines of evidence suggest a strong association between chronic inflammation and increased susceptibility to neoplastic transformation and cancer development. It has been estimated that up to $20 \%$ of all tumours arise from conditions of persistent inflammation such as chronic infections or autoimmune diseases. A direct link between these two conditions has been established in particular in the gastrointestinal tract $(1,2)$.

The association of autoimmune thyroiditis (AIT) and thyroid cancer was first documented in 1955 (3). Since that time the coexistence of AIT and papillary thyroid cancer (PTC) has been well documented in the literature. Some authors believe that coexistent thyroiditis is associated with lower tumour stage and better prognosis of thyroid cancer (3-8). Whether this relationship is causal or merely fortuitous remains a point of dispute. AIT is one of the most common autoimmune diseases, the most common inflammation of the thyroid and the most common cause of hypothyroidism in the industrialized world. Developed countries with sufficient iodine intake due to iodine prophylaxis have been facing an 'epidemic' of this disease. Similarly, PTC has been recognized as a cancer with the highest increasing incidence during last three decades (9). AIT and PTC seem to show a stronger association than expected by simple probabilistic explanations. Pathogenic immunobiological links between them are possible. The results of many studies exploring this issue can be clustered in three groups: i) AIT is induced as a response to a pre-existing PTC, ii) PTC is induced or facilitated by a pre-existing chronic inflammatory process, iii) common mechanisms are responsible for both diseases (10). 
The ability of thyroid cancer to produce an immune response is caused by the RET/PTC oncogene induction of a pro-inflammatory transcriptional program. Oncogenes responsible for cell neoplastic transformation elicit an inflammatory pro-tumourigenic microenvironment. Pro-inflammatory molecules, such as cytokines and chemokines, produced by immune infiltrates, contribute to the regulation of cellular processes for cancer onset and progression, for tumour cell proliferation, angiogenesis and metastases $(1,2,6)$.

Alternatively, chronic inflammation may enhance carcinogenesis by promoting genomic instability (11). Molecular studies showed that thyrocytes treated with IL-1beta and TNF-alpha were able to induce cyclooxygenase-2 (COX-2) and secrete IL-6 (10). Elevated COX-2 expression is known to be associated with the carcinogenesis of various types of neoplasms, by both inhibiting apoptosis and promoting angiogenesis. COX-2 expression was found to be in thyroid cancers and thyroid epithelium from AITs, but not in normal thyroid. This finding may provide a basis for a relationship between carcinogenesis and autoimmunity (12). Molecular studies have identified activation of the RET/PTC rearrangement-induced MAPK signalling pathway as the driving force in the development of PTC in the context of AIT (1).

If AIT could be recognized as a precursor or a risk condition for PTC, or at least for a subset of PTC, this would have an obvious high clinical importance, given that AIT is a very common disease in many countries.

Insulin-like growth factor-I (IGF-1) might also play an important role in development of thyroid carcinoma due to its mitogenic and anti-apoptotic properties (13). Complement component $\mathrm{C} 3$ (C3) has been found overexpressed in numerous cancer tissues, such as oesophageal cancer, gastric cancer, and lung cancer as well as in PTC tissue when compared to normal tissue (14).

Epidemiological data report a strong female predisposition for thyroid cancer. Female predominance of thyroid cancer in the childbearing period suggests that oestrogen and progesterone may play vital roles in the pathogenesis of thyroid neoplasms (15).

Therefore, the aim of this study was to investigate possible associations of AIT and PTC by assessing mRNA levels expressed from key genes. The mRNA expression pattern of nuclear thyroid hormone receptors (TR), retinoid/rexinoid receptors ( $R X R$ ), vitamin $D_{3}$ receptor, progesterone receptor, selected co-repressors, type I iodothyronine 5'-deiodinase (DIO1), proliferation markers (MKi67, PCNA), insulin-like growth factor 1 (IGF-1), anti- and proapoptotic genes (Bcl2, BAX, p53), complement C3 mRNA was compared in thyroid tumour tissue of PTCs without AIT (PTC/AIT-) and with coexisting AIT (PTC/AIT+)in order to find whether expression of selected genes may take part in the progression of thyroid malignancies.

\section{Materials and methods}

Clinical samples. Tumour and surrounding uninvolved thyroid tissue were collected from 33 unselected PTC patients (six male subjects and twenty-seven female subjects) at the St. Elisabeth Cancer Institute in Bratislava, Slovakia, whose surgery was planned by physicians who were not connected to this study.
Collected tumour tissues and peritumoural thyroid tissue from 14 patients with co-existent AIT [PTC/AIT+, and N+ (7 out of 14); respectively] and 19 patients without AIT [PTC/AIT-, and N- (10 out of 19); respectively] were immediately frozen in liquid nitrogen and stored at $-70^{\circ} \mathrm{C}$. Tumours were classified and the clinical stage determined by the tumour, node, metastasis (TNM) system (16) and the presence of AIT, based on histological evaluation. The study was approved by the Ethics Committee of the St. Elisabeth Cancer Institute in Bratislava, Slovakia and unambiguously conducted according to the principles of the Declaration of Helsinki. Consent was obtained from each patient or subject after full explanation of the purpose and nature of all study procedures.

Reverse transcription-semiquantitative polymerase chain reaction. Total RNA was isolated using TRI Reagent ${ }^{\circledR}$ (Molecular Research Center, Inc, Cincinnati, USA) according to the manufacturer's instructions. The concentration of RNA was determined by spectrophotometry at $260 \mathrm{~nm}$ and the purity assessed from the ratio of absorbance, A260/A280 nm, using a NanoDrop 2000 spectrophotometer (Thermo Scientific, Germany). Reverse transcription (RT) was performed with $2 \mu \mathrm{g}$ of total DNAse I-treated (Thermo Scientific, Germany) RNA and the Ready-to-Go You-Prime First-Strand Beads (Amersham Pharmacia Biotech, Inc., USA) according to the manufacturer's protocol.

Semiquantitative real-time PCR was performed in duplicates in a total volume of $20 \mu 1$ using SensiFAST ${ }^{\mathrm{TM}}$ SYBR Hi-Rox Kit (Bioline, Great Britain) and $0.25 \mu \mathrm{M}$ of each primer and RT product: $10 \mathrm{ng}$ (RPS18) and $30 \mathrm{ng}$ (for the other genes). Amplification and detection were performed with an ABI Prisma 7900HT detection system (Applied Biosystems, USA) under the following conditions: $95^{\circ} \mathrm{C}$ for $2 \mathrm{~min}, 40$ cycles of denaturation $\left(95^{\circ} \mathrm{C}, 5 \mathrm{sec}\right)$ and annealing $(30 \mathrm{sec})$, and the final melting curve analysis. The data are expressed using the $2^{-\Delta \Delta \mathrm{Cq}}$ method as the relative level of each mRNA normalized to that of the housekeeping gene RPS18. The oligonucleotide of the primers employed in this study along with the corresponding annealing times are summarized in Table I (17). These conditions were proven to be in the log phase for each amplified sequence. Triton tumour tissue and $\mathrm{LNCaP}$ prostatic cancer cell line were used as a positive control (18). A negative control without cDNA template was run with every assay batch in order to assess overall specificity.

Statistical analysis. Data are expressed as medians (range $5-95 \%$ ) of two PCR analyses. Differences between more than two groups were assessed by one-way analysis of variance followed by Bonferroni post hoc test using SigmaPlot ${ }^{\circledR} 11.0$ (Systat Software $\mathrm{GmbH}$ ). $\mathrm{P}<0.05$ was considered to indicate a statistically significant difference.

\section{Results}

Six male subjects and twenty-seven female subjects were enrolled in this study. The mean age of patients at surgery was $49.91 \pm 17.06$ years (mean \pm SD). Clinicopathological parameters of the 33 cases of PTC are presented in Table II. Among 33 patients, there were 14 patients with co-existent AIT (PTC/AIT+) and 19 patients without AIT (PTC/AIT-). The 
Table I. Primers for semiquantitative PCR.

\begin{tabular}{llr} 
Gene & \multicolumn{1}{c}{ Sequence (5'-3') } & Annealing te \\
\hline RAR $\alpha$ & F: ACCCCCTCTACCCCGCATCTACAAG and R: CATGCCCACTTCAAAGCACTTCTGC & 60 \\
RAR $\beta$ & F: ATTCCAGTGCTGACCATCGAGTCC and R: CCTGTTCTGTGTCATCCATTTCC & 62 \\
RAR $\gamma$ & F: TACCACTATGGGGTCAGC and R: CCGGTCATTTCGCACAGCT & 60 \\
RXR $\alpha$ & F: CTTTTGTTCCGTGCTGTTTA and R: CTGAGGTCTTTGCTGATGACAC & 60 \\
RXR $\beta$ & F: TACAGGGCAGAACCAAGAACA and R: ATGAGGCAAGATGAGAAGGAAG & 60 \\
RXR $\gamma$ & F: AGAAAGACAGAGGAGCCGAGA and R: CAGAGAAGTGGGGAATACGC & 60 \\
RPS18 & F: TCTAGTGATCCCTGAAAAGTTCC and R: CGTGGATTCTGCATAATGGTG & 60 \\
TR $\alpha$ & F: AGGAGAACAGTGCCAGGTCA and R: TCTTGAAGCGGCACAGCTGG & 60 \\
TR $\beta$ & F: AACTACAGGTATAAGGCTGATTCAC and R: ATGCTTCTCTGCGTATATGCC & 60 \\
VDR & F: GACTTTGACCGGAACGTGCGG and R: CATCATGCCGATGTCCACACA & 60 \\
PR & F: TCTATTCATTATGCCTTACCATGTG and R: AACCAATTGCCTTGATGAGC & 60 \\
SMRT & F: TGTGGTTCATAAGCCATCTGC and R: AATCTTCCCCTCCTCCC & 60 \\
N-CoR & F: AGCATTCCATCCCTACGGG and R: TGGACCCCTTCACCAAAG & 60 \\
IGF-1 & F: TGACTCCACTTCCTCTAACTCCA and R: AAACCTCTCACCTCAACCTCA & 60 \\
C3 & F: TGCGGCTACCCTACTCTGTTGTTCG and R: GACGGCAGCCTTGACTTCCACTTCC & 60 \\
PCNA & F: AGTGGAGAACTTGGAAATGGAA and R: GAAGAGAGTGGAGTGGCTTTTG & 60 \\
MKi6 & F: TCAGAAAGGGAAAGGAGAAGC and R: GACACACACATTGTCCTCAGC & 60 \\
Bc12 & F: GACTTCGCCGAGATGTCCAG and R: CAGGTGCCGGTTCAGGTACT & 60 \\
BAX & F: TGCTTCAGGGTTTCATCCAGGA and R: ACGGCGGCAATCATCCTCTC & 60 \\
p53 & F: CCCCTCCTGGCCCCTGTCATCTTCT and R: GCAGCGCCTCACAACCTCCGTCAT & 60 \\
DIO1 & F: GGACATCAGAAATCACCAGA and R: TTCCTCTGGGTTGTAGTTCC & 58
\end{tabular}

F, forward; R, reverse

mean age of patients with AIT was $45.08 \pm 16.33$ and without AIT $39.85 \pm 17.63$ years (mean \pm SD). Histologically confirmed lymph node metastases were present in 16 patients.

We investigated the mRNA expression patterns of selected nuclear receptors and other molecular targets in the PTC tissue and peritumoural tissue of patients without AIT (PTC/AIT-, and $\mathrm{N}$-; respectively) and compared them to those with coexisting AIT (PTC/AIT+, and N+; respectively).

Significantly decreased expression levels of both TRalpha and TRbeta mRNA in PTC/AIT+ tumour tissue was seen when compared to tumour tissue of PTC/AIT- patients (Fig. 1A and B). A similar decrease of TRbeta mRNA (but not TRalpha mRNA) was also detected in non-tumour thyroid tissues of PTC/AIT+ patients in comparison to non-tumour tissues of PTC/AIT- patients.

We found significantly decreased levels of RARalpha mRNA in thyroid tumour tissue $(\mathrm{P}<0.05)$ of patients with AIT (group PTC/AIT+) compared to the tumour tissue of patients without AIT (group PTC/AIT-; Fig. 2A). A similar effect of AIT was found on the significantly decreased expression of RARgamma mRNA in thyroid tumour tissue of PTC/AIT+ patients (Fig. 2B).

There was no significantly changed expression of RARbeta mRNA in the thyroid tumour tissue between patients without or with AIT; however, there were significantly $(\mathrm{P}<0.05)$ reduced RARbeta mRNA levels in non-tumour thyroid tissue of the PTC/AIT+ subgroup when compared to non-tumour tissue of PTC/AIT- patients and there were significantly higher levels of RXRgamma mRNA in thyroid tumour tissue compared to non-tumour thyroid tissue of both PTC/AIT+ and PTC/AIT- patient subgroups (data not shown). We did not find any significant differences in expression of RXRalpha, RXRbeta, co-repressors NCoR and SMRT mRNAs in tumour tissues of PTC/AIT-, compared to tumour tissues of PTC/AIT+ patients (data not shown). We found significantly increased expression of dihydroxyvitamin $\mathrm{D}_{3}$ receptors (VDR) mRNA in tumour tissue of PTC/AIT+ when compared to tumour tissue of PTC/AIT- patients (Fig. 3A). On the other hand, the levels of PR mRNA were decreased in tumour tissue of PTC/AIT+ patients compared to expression in tumours of patients without AIT (Fig. 3B).

As shown in Fig. 4A, we found that there was either absent or significantly lower expression of type I iodothyronine 5'-deiodinase (DIO1) in tumour tissue compared to non-tumour thyroid tissue in both of patients and there were not any significant differences between PTC/AIT- and PTC/AIT+ patients.

We sought to investigate the question whether the process of AIT might take part in the proliferation and apoptosis in both tumour and non-tumour thyroid tissue. To study proliferation, two well-known markers, MKi67 and PCNA were evaluated, and significantly increased expression level of MKi67 mRNA was found in tumour tissue of PTC/AIT+ when compared to PTC/AIT- (Fig. 4B); however, the expression of PCNA marker remained unchanged (data not shown). We found that tumours from the PTC/AIT+ patients showed significantly decreased levels of IGF-1 mRNA compared to expression in tumours 
Table II. Clinicopathological parameters of the 33 cases of PTC.

\begin{tabular}{|c|c|c|c|c|}
\hline Patient & Gender & Age, years & AIT status & Histology \\
\hline $\mathrm{P} 1$ & $\mathrm{~F}$ & 20 & PTC/AIT- & T1bN0M0 \\
\hline $\mathrm{P} 2$ & $\mathrm{~F}$ & 69 & PTC/AIT- & T3N0M0 \\
\hline P3 & M & 31 & PTC/AIT- & T1bN1aM0 \\
\hline P4 & $\mathrm{F}$ & 32 & PTC/AIT- & T1bN0M0 \\
\hline P5 & $\mathrm{F}$ & 67 & PTC/AIT- & T1aNxM0 \\
\hline P6 & $\mathrm{F}$ & 34 & PTC/AIT- & T1aN1aM0 \\
\hline P7 & $\mathrm{F}$ & 37 & PTC/AIT- & T3N1bM0 \\
\hline P8 & M & 23 & PTC/AIT- & T3N1aM0 \\
\hline P9 & $\mathrm{F}$ & 34 & PTC/AIT- & T3N1bM0 \\
\hline P10 & $\mathrm{F}$ & 69 & PTC/AIT- & T3N0M0 \\
\hline P11 & $\mathrm{F}$ & 39 & PTC/AIT- & T3N0M0 \\
\hline P12 & $\mathrm{F}$ & 20 & PTC/AIT- & T1bN0M0 \\
\hline P13 & $\mathrm{F}$ & 38 & PTC/AIT- & T1aN0M0 \\
\hline P14 & $\mathrm{F}$ & 69 & PTC/AIT- & T2N0M0 \\
\hline P15 & M & 19 & PTC/AIT- & $\mathrm{T} 2 \mathrm{~N} 1 \mathrm{Mx}$ \\
\hline P16 & $\mathrm{F}$ & 38 & PTC/AIT- & T1bN1aM0 \\
\hline P17 & $\mathrm{F}$ & 38 & PTC/AIT- & T3N1bM0 \\
\hline P18 & M & 38 & PTC/AIT- & T3N1aM0 mikro \\
\hline P19 & M & 62 & PTC/AIT- & T1aN1bM0 \\
\hline P20 & $\mathrm{F}$ & 20 & PTC/AIT- & T1bN0M0 \\
\hline P21 & $\mathrm{F}$ & 36 & PTC/AIT+ & T3N0M0 \\
\hline $\mathrm{P} 22$ & $\mathrm{~F}$ & 39 & PTC/AIT+ & T1bN1aM0 \\
\hline $\mathrm{P} 23$ & $\mathrm{~F}$ & 33 & PTC/AIT+ & T1bN0M0 \\
\hline P24 & $\mathrm{F}$ & 79 & PTC/AIT+ & T1bN0M0 \\
\hline $\mathrm{P} 25$ & $\mathrm{~F}$ & 47 & PTC/AIT+ & T3N1M0 \\
\hline P26 & M & 44 & PTC/AIT+ & T3N1bM0 \\
\hline P27 & $\mathrm{F}$ & 43 & PTC/AIT+ & T3aN1bM0 \\
\hline P28 & $\mathrm{F}$ & 26 & PTC/AIT+ & T1aN1aM0 \\
\hline P29 & $\mathrm{F}$ & 70 & PTC/AIT+ & T1bN0M0 \\
\hline P30 & $\mathrm{F}$ & 34 & PTC/AIT+ & T1aN1aM0 \\
\hline P31 & $\mathrm{F}$ & 66 & PTC/AIT+ & T3N1M0 \\
\hline P32 & $\mathrm{F}$ & 36 & PTC/AIT+ & T3N0M0 \\
\hline P33 & $\mathrm{F}$ & 33 & PTC/AIT+ & T1bN0M0 \\
\hline
\end{tabular}

F, female; M, male; PTC, papillary thyroid carcinoma; AIT, autoimmune thyroiditis.

from the PTC/AIT- patients. A similar pattern was demonstrated in corresponding non-tumour thyroid tissue (Fig. 4C). No differences were found in the expression of anti-apoptotic gene Bcl2 and pro-apoptotic genes BAX and p53 (data not shown). Significantly increased levels of complement C3 mRNA in tumour tissue of PTC/AIT+ patients were detected compared to the tumour tissue of PTC/AIT- patients (Fig. 4D).

\section{Discussion}

Papillary thyroid cancer (PTC) is the most frequent thyroid cancer histotype, accounting for more than $80 \%$ of all thyroid malignancies (19). Previously, a meta-analysis of the literature on Hashimoto's thyroiditis and PTC risk was conducted in order to investigate the question of whether AIT may predispose patients to the development of PTC (20). Consequently, we sought to investigate selected molecular targets in PTC comparing those cases without AIT to those with coexisting AIT.

It has been demonstrated that large amounts of IL-1beta are present in AIT. IL-1beta is a proinflammatory cytokine and mediates induction of Fas on thyroid follicular cells, which result in apoptotic tissue damage (21). IL-1beta can activate MAP kinases and the NF- $\kappa \mathrm{B}$ pathway. IL-1beta was found to modulate the retinoid signal transduction pathway, and IL-1beta gene is a direct, downstream target gene of retinoic acid thus it may indirectly regulate cell growth (22). Moreover, IL-1beta can block the insulin and insulin-like growth factor pathways by inhibiting the receptor kinase activity. Furthermore, IGF-1 suppresses apoptosis and induces thyroid proliferation via protein-tyrosine kinase-dependent signalling pathway (23). Moreover, IGF-1 and oestrogen can act synergistically through the IGF-1 signalling cascade (24). Previous findings indicate that cross-talk between retinoic acids, thyroid hormone and oestrogens pathways acting through nuclear receptors plays important roles in the regulation of many processes in various tissues and it has been suggested that cancer progression may be associated with alteration in metabolism and/or signalling pathways of these components (25).

Nuclear receptors (NRs)-ligand-inducible transcription factors, members of the nuclear receptor superfamily have been at the forefront of cancer research, where they are known to act as critical regulators of cancer diseases and are also playing a crucial role as biomarkers for tumour subclassification and predominantly targets for hormone therapy (26). Molecular endocrinology approaches for studying nuclear receptor superfamily members clearly demonstrate the importance of transcription factors inducible by biologically active molecules or hormones in the biology and possible clinical treatment of thyroid cancer. Since no relevant data on nuclear receptors expression showing associations of autoimmune thyroiditis and PTC do exist, our data thus represent the first insight into the processes, where the starting points exist at the nuclear receptors level.

Thyroid hormone receptors (TRalpha, TRbeta) are ligand-inducible transcription factors that mediate a variety of the genomic actions of 3,5,3'-triiodothyronine. Loss of normal functions of TRs by deletion, reduced expression, or by mutations could contribute to cancer development, progression and metastasis (27). Our data showing a marked diminution of TRalpha and TRbeta mRNA expression in PTC tumours in comparison with non-tumour thyroid tissues and additional decreases in expression of both TR in PTC with coexisting AIT supports the hypothesis suggesting that the loss or marked decrease of TR may contribute to cancer development and progression.

Several case studies have noted the development of AIT in patients following or within the last few weeks of isotretinoin treatment (28). Moreover, retinoid receptor (RAR) and retinoid $\mathrm{X}$ receptors (RXR) subtypes are differentially expressed in thyroid cancer and in thyroid carcinoma cell lines when compared to non-tumour thyroid tissue and cells $(29,30)$. A comparison of thyroid tumours and case-matched normal thyroid tissue confirmed different tumour expression of 


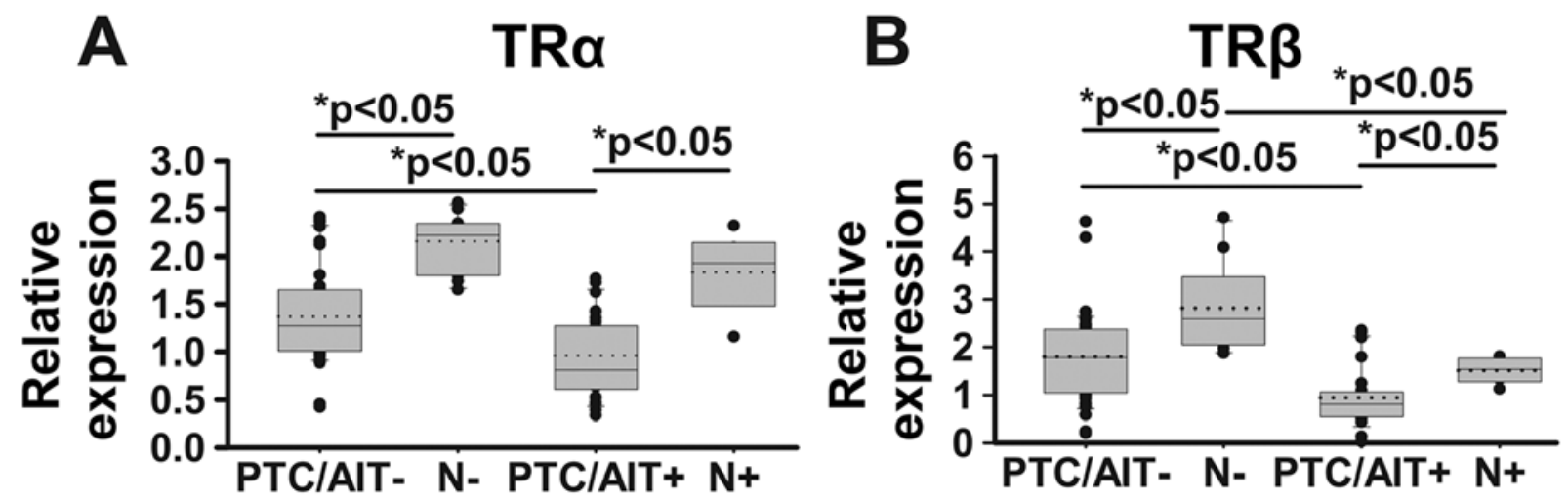

Figure 1. Relative levels of (A) TR $\alpha$ and (B) TR $\beta$ mRNA in the tumour and normal tissues of the patients with or without coexisting AIT. PTC/AIT- (n=19) and PTC/AIT+ $(n=14)$ represents papillary thyroid carcinomas with and without autoimmune thyroiditis, respectively. $\mathrm{N}$ - represents peritumoural thyroid tissue; $\mathrm{N}+$ represents peritumoural thyroid tissues with autoimmune thyroiditis. Data are expressed as the medians (range 5-95\%) of two PCR analyses. Differences between $>2$ groups were assessed by one-way analysis of variance followed by the Bonferroni. "P $<0.05$, as indicated. TR, thyroid hormone receptor; AIT, autoimmune thyroiditis; PTC, papillary thyroid carcinoma.
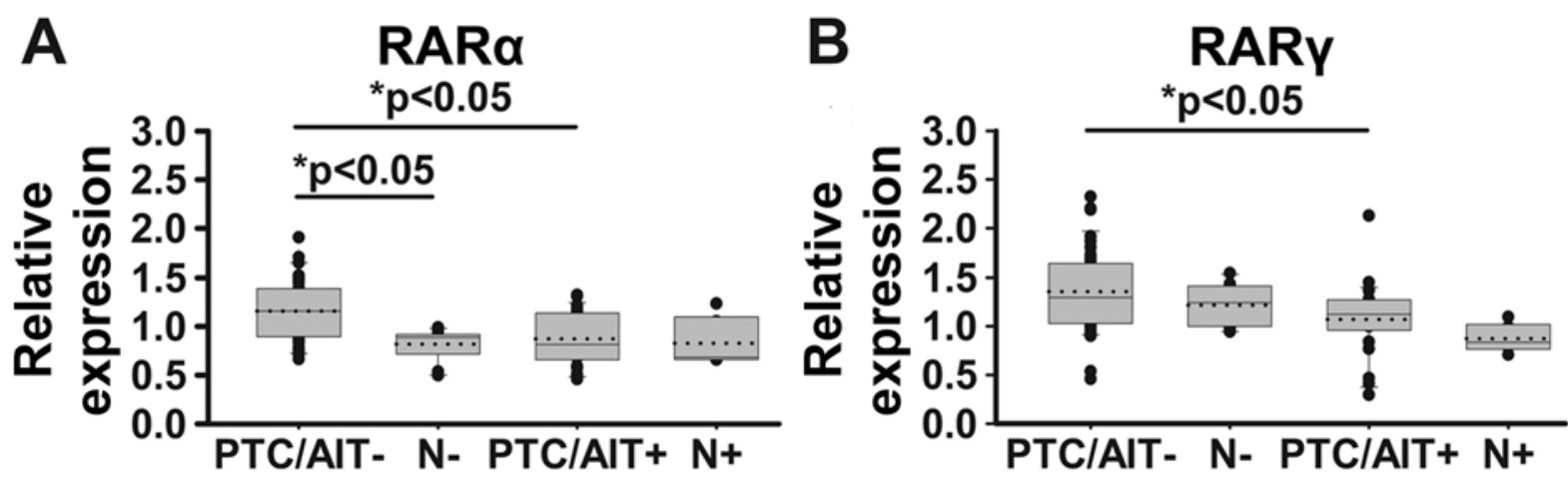

Figure 2. Relative levels of (A) RAR $\alpha$ and (B) RAR $\gamma$ mRNA in the tumour and normal tissues of the patients with or without coexisting AIT. PTC/AIT- (n=19) and PTC/AIT+ $(n=14)$ represents papillary thyroid carcinomas with and without autoimmune thyroiditis, respectively. N- represents peritumoural thyroid tissue; $N+$ represents peritumoural thyroid tissues with autoimmune thyroiditis. Data are expressed as the medians (range 5-95\%) of two PCR analyses. Differences between $>2$ groups were assessed by one-way analysis of variance followed by the Bonferroni. "P $<0.05$, as indicated. RAR, retinoid receptors; AIT, autoimmune thyroiditis; PTC, papillary thyroid carcinoma.
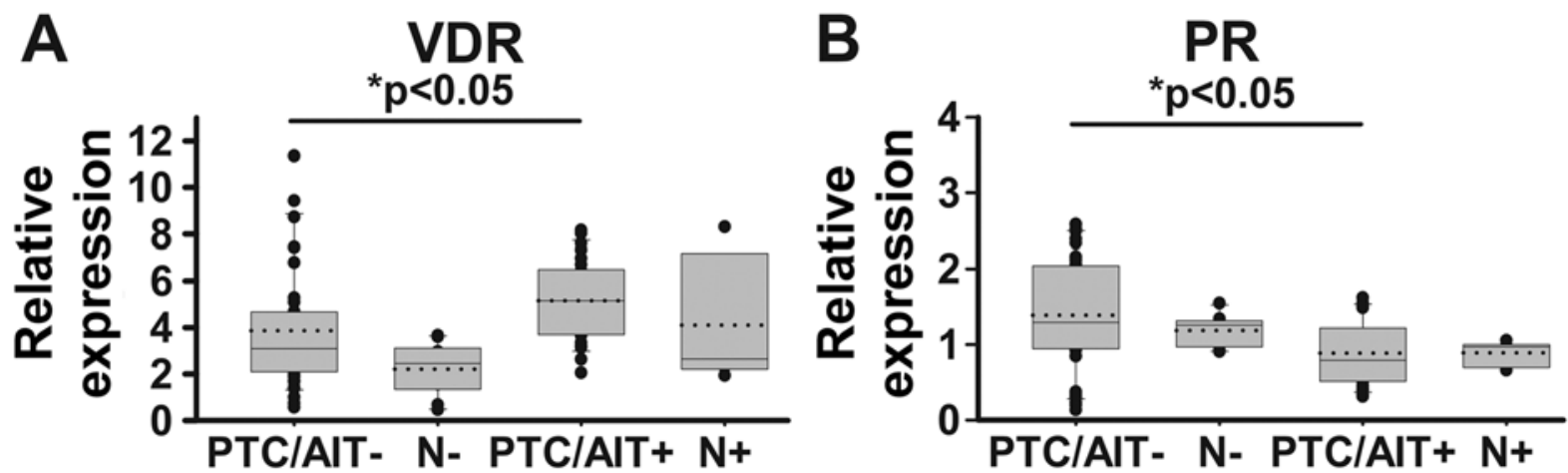

Figure 3. Relative levels of (A) VDR and (B) PR mRNA in the tumour and normal tissues of the patients with or without coexisting AIT. PTC/AIT- (n=19) and PTC/AIT+ $(n=14)$ represents papillary thyroid carcinomas with and without autoimmune thyroiditis, respectively. N- represents peritumoural thyroid tissue; $\mathrm{N}+$ represents peritumoural thyroid tissues with autoimmune thyroiditis. Data are expressed as the medians (range 5-95\%) of two PCR analyses. Differences between $>2$ groups were assessed by one-way analysis of variance followed by the Bonferroni. ${ }^{*} \mathrm{P}<0.05$, as indicated. VDR, dihydroxyvitamin $\mathrm{D}_{3}$ receptors; PR, progesterone receptors; AIT, autoimmune thyroiditis; PTC, papillary thyroid carcinoma.

RARalpha, RARgamma, and missing or highly significant decreases of RXRgamma expression in intact thyroid tissue (17,31). Here, our data demonstrates a similarly marked diminution of RARalpha and RARgamma mRNA expression in PTC with coexisting AIT when compared to PTC without AIT. Thus, data on differences in RAR or RXR subtype expression may have a valuable impact for the differential diagnosis of thyroid neoplasms (30). 
A

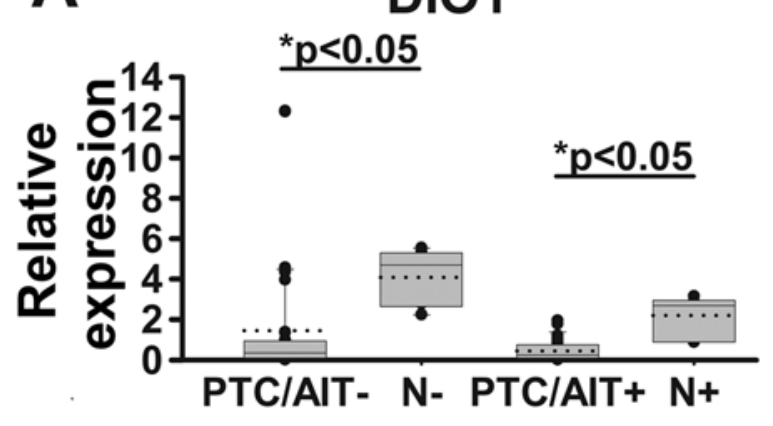

C

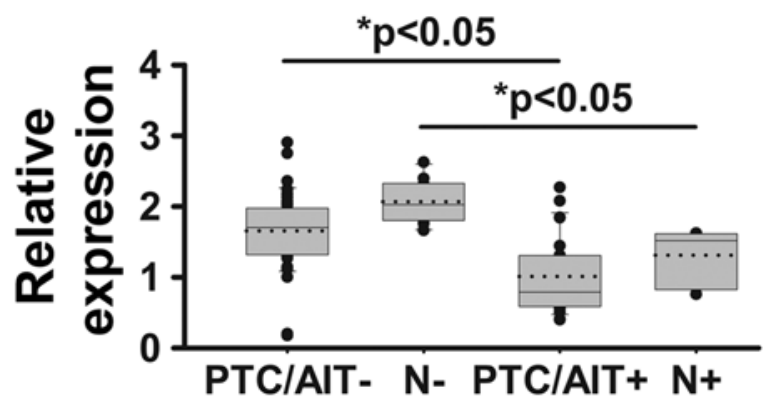

B

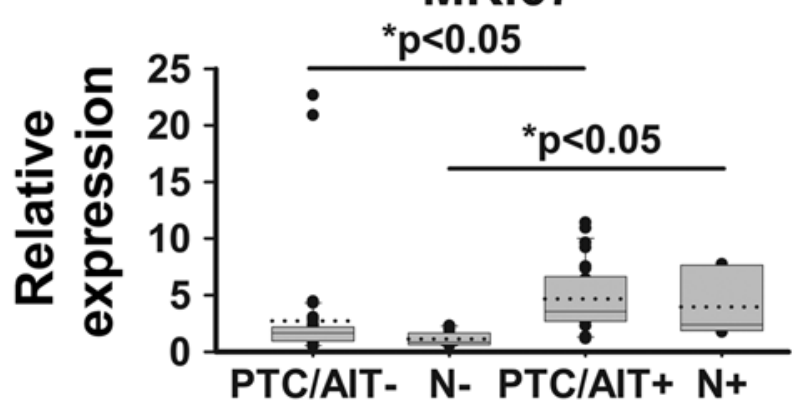

D

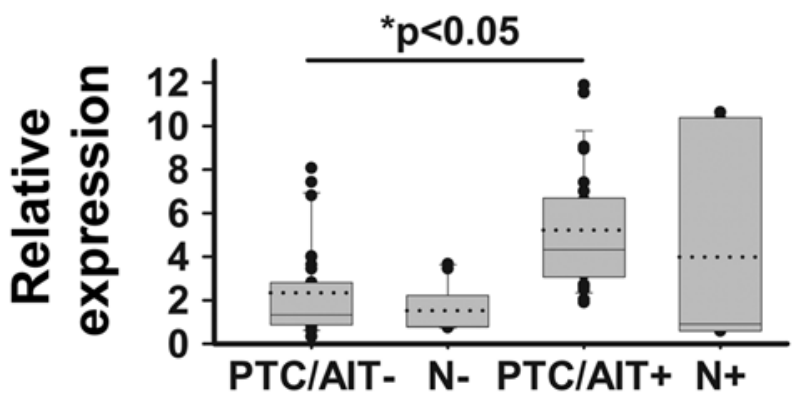

Figure 4. Relative levels of (A) DIO1, (B) MKi67, (C) IGF-1 and (D) complement C3 mRNA in the tumour and normal tissues of the patients with or without coexisting AIT. PTC/AIT- $(n=19)$ and PTC/AIT+ $(n=14)$ represents papillary thyroid carcinomas with and without autoimmune thyroiditis, respectively. $\mathrm{N}$ - represents peritumoural thyroid tissue; $\mathrm{N}+$ represents peritumoural thyroid tissues with autoimmune thyroiditis. Data are expressed as the medians (range $5-95 \%$ ) of two PCR analyses. Differences between $>2$ groups were assessed by one-way analysis of variance followed by the Bonferroni. $\mathrm{P}<0.05$, as indicated. DIO1, type I iodothyronine 5'-deiodinase; MKi67, marker of proliferation Ki-67; IGF-1, insulin-like growth factor 1; AIT, autoimmune thyroiditis; PTC, papillary thyroid carcinoma.

Recent evidence has shown that vitamin D deficiency could be associated with autoimmune thyroid diseases such as Hashimoto's thyroiditis and Graves' disease, and there is impaired vitamin D signalling in thyroid tumours (32). The VDR was found to be expressed in both normal and malignant thyroid tissue. Moreover, VDR and CYP27B1 expressions were both increased in papillary thyroid cancer when compared to normal thyroid tissue (reviewed in 26). Our data clearly demonstrates significant enhancement of the VDR mRNA expression in PTC with coexisting AIT as compared to PTC without AIT. The present study shows that malignant cells in PTC express progesterone receptor (PR), thus opening the door for further investigations to determine whether those patients could benefit from hormonal therapy. Oestrogen receptors seem to have a role in the metastatic process of PTC, as they are expressed more in the metastatic tumours than in the primary tumours (15).

Type I, iodo-L-thyronine 5'-deiodinase (DIO1) is a selenoezyme responsible for monodeiodination of L-thyroxine to biologically active 3,5,3'-triiodothyronine, the cognate TR ligand. DIO1 activity was found to be decreased in PTC, and it is conceivable that understanding how deiodinase(s) dysregulation in thyroid tumour cells affects thyroid hormone signalling would relate to tumour progression could lead to new antineoplastic approaches (33). In spite of the fact that our data corresponded to the referenced findings, no significant difference has been found in the mRNA expression of DIO1 between PTC- and PTC+ patients. The Ki-67 protein (also known as MKi67) is a cellular marker that is strongly associated with cell proliferation. Our data has clearly shown significantly increased MKi67 mRNA expression in PTC with coexisting AIT compared to PTC without AIT, suggesting increased cell proliferation in the PTC patients with AIT. Insulin-like growth factor-I (IGF-I) is a protein with mitogenic and anti-apoptotic properties (23), and high circulating concentrations have been shown to be associated with an increased risk of developing cancer at several sites, such as breast, prostate, and colorectal cancer (34). Recently, it has been suggested that IGF-I concentrations may be positively associated with risk of differentiated thyroid carcinoma (13). $\mathrm{C} 3$ is a central protein of the complement system, playing a crucial role in the activation of the complement system. $\mathrm{C} 3$ has been found to be overexpressed in numerous cancer tissues as well as in PTC tissue when compared to corresponding non-neoplastic tissues $(14,35)$. Our data clearly demonstrated significant enhancement of C3 mRNA expression in PTC with coexisting AIT compared to PTC without AIT.

In conclusion, the expression of investigated molecular targets by mRNA level clearly demonstrated significant differences in PTC with coexisting AIT compared to PTC without AIT. Based on these novel findings, we suggest that AIT is a predisposing factor to the development of PTC. To better understand mechanisms underlying this association, further studies at the molecular level are needed. Consideration of the possibility that AIT may be a predisposing cause of PTC means that it may be necessary to study the causes of the high prevalence of AIT and to investigate whether reduction 
of exogenous factors that contribute to the development of thyroid autoimmunity may be of value.

\section{Acknowledgements}

The authors would like to thank Professor Kenneth B. Ain (Thyroid Oncology Program, University of Kentucky Medical Center, USA) for editing the language of the manuscript.

\section{Funding}

The present study was supported by grants from the APVV grant agency (grant nos. APVV-15-0372 and APVV-0160-11) and the VEGA grant (grant no. 2/0171/17).

\section{Availability of data and materials}

All data generated or analysed during this study are included in this published article.

\section{Authors' contributions}

DM, JP, MG and JB conceived and designed the experiments. DM and LT conducted the PCR experiments and analysed the data. KK and KM performed histological analyses. DM, JP and $\mathrm{JB}$ reviewed the final results and drafted the paper.

\section{Ethics approval and consent to participate}

The present study was approved by the Ethics Committee of the St. Elisabeth Cancer Institute in Bratislava, Slovakia and was conducted according to the principles of the Declaration of Helsinki. Written informed consent was obtained from each patient/subject.

\section{Patient consent for publication}

Consent for the publication of the clinical and pathological data was obtained from all patients who were involved in the present study.

\section{Competing interests}

The authors declare that they have no competing interests.

\section{References}

1. Bozec A, Lassalle S, Hofman V, Ilie M, Santini J and Hofman P. The thyroid gland: A crossroad in inflammation-induced carcinoma? An ongoing debate with new therapeutic potential. Curr Med Chem 17: 3449-3461, 2010.

2. Muzza M, Degl'Innocenti D, Colombo C, Perrino M, Ravasi E, Rossi S, Cirello V, Beck-Peccoz P, Borrello MG and Fugazzola L: The tight relationship between papillary thyroid cancer, autoimmunity and inflammation: Clinical and molecular studies. Clin Endocrinol (Oxf) 72: 702-708, 2010

3. Babli S, Payne RJ, Mitmaker E and Rivera J: Effects of chronic lymphocytic thyroiditis on the clinicopathological features of papillary thyroid cancer. Eur Thyroid J 7: 95-101, 2018.

4. Ott RA, Calandra DB, McCall A, Shah KH, Lawrence AM and Paloyan E: The incidence of thyroid carcinoma in patients with Hashimoto's thyroiditis and solitary cold nodules. Surgery 98: 1202-1206, 1985.
5. Schäffer A, Palitzsch KD, Seiffarth C, Hohne HM, Riedhammer FJ, Hofstädter F, Schölmerich J and Rüschoff J: Coexistent thyroiditis is associated with lower tumour stage in thyroid carcinoma. Eur J Clin Invest 28: 838-844, 1998.

6. Loh KCH, Greenspan FS, Dong F, Miller TR and Yeo PPB: Influence of lymphocytic thyroiditis on the prognostic outcome of patients with papillary thyroid carcinoma. J Clin Endocrinol Metab 84: 458-463, 1999.

7. Shih ML, Lee JA, Hsieh CB, Yu JC, Liu HD, Kebebew E, Clark OH and Duh QY: Thyroidectomy for Hashimoto's thyroiditis: Complications and associated cancers. Thyroid 18: 729-734, 2008.

8. Fiore E, Rago T, Latrofa F, Provenzale MA, Piaggi P, Delitala A, Scutari M, Basolo F, Di Coscio G, Grasso L, et al: Hashimoto's thyroiditis is associated with papillary thyroid carcinoma: Role of TSH and of treatment with L-thyroxine. Endocr Relat Cancer 18: 429-437, 2011.

9. Vaccarella S, Franceschi S, Bray F, Wild CP, Plummer M and Dal Maso L: Worldwide thyroid-cancer epidemic? The increasing impact of overdiagnosis. N Engl J Med 7: 614-617, 2016.

10. Antonaci A, Consorti F, Mardente S and Giovannone G: Clinical and biological relationship between chronic lymphocytic thyroiditis and papillary thyroid carcinoma. Oncol Res 17: 495-503, 2009.

11. Prescott SM and Fitzpatrick FA: Cyclooxygenase-2 and carcinogenesis. Biochim Biophys Acta 1470: M69-M78, 2000.

12. Cornetta A, Russell JP, Cunnane M, Keane WM and Rothstein JL: Cyclooxygenase-2 expression in human thyroid carcinoma and Hashimoto's thyroiditis. Laryngoscope 112: 238-242, 2002.

13. Schmidt JA, Allen NE, Almquist M, Franceschi S, Rinaldi S, Tipper SJ, Tsilidis KK, Weiderpass E, Overvad K, Tjønneland A, et al: Insulin-like growth factor-i and risk of differentiated thyroid carcinoma in the European prospective investigation into cancer and nutrition. Cancer Epidemiol Biomarkers Prev 23: 976-985, 2014.

14. Yu J, Mai W, Ciu Y and Kong L: Key genes and pathways predicted in papillary thyroid carcinoma based on bioinformatics analysis. J Endocrinol Invest 39: 1285-1293, 2016.

15. Eldien MMS, Abdou AG, Rageh T, Abdelrazek E and Elkholy E: Immunohistochemical expression of ER- $\alpha$ and PR in papillary thyroid carcinoma. Ecancermedicalscience 11: 748, 2017.

16. Brierley JD, Gospodarowicz MK and Wittekind C (eds): TNM classification of malignant tumours. 8th edition, Oxford, Wiley Blackwell, ISBN 9781119263579, 2017.

17. Brtko J, Sejnová D, Ondková S and Macejová D: Malignant Triton tumour exhibits a complete expression pattern of nuclear retinoid and rexinoid receptor subtypes. Gen Physiol Biophys 28: 425-427, 2009.

18. Macejová $\mathrm{D}$, Galbavý $\mathrm{S}$, Podoba J, Bialešová L and Brtko J: mRNA expression pattern of retinoic acid and retinoid $\mathrm{X}$ nuclear receptor subtypes in human thyroid papillary carcinoma. Oncol Rep 30: 2371-2378, 2013.

19. Franceschi S, Boyle P, Maisonneuve P, La Vecchia C, Burt AD, Kerr DJ and MacFarlane GJ: The epidemiology of thyroid carcinoma. Crit Rev Oncog 4: 25-52, 1993.

20. Lai X, Xia Y, Zhang B, Li J and Jiang Y: A meta-analysis of Hashimoto's thyroiditis and papillary thyroid carcinoma risk. Oncotarget 8: 62414-62424, 2017.

21. Paolieri F, Salmaso C, Battifora M, Montagna P, Pesce G, Bagnasco M, Richiusa P, Galluzzo A and Giordano C: Possible pathogenetic relevance of interleukin-1 beta in 'destructive' organ-specific autoimmune disease (Hashimoto's thyroiditis). Ann N Y Acad Sci 876: 221-228, 1999.

22. Liu L and Gudas LJ: Retinoic acid induces expression of the interleukin-1beta gene in cultured normal human mammary epithelial cells and in human breast carcinoma lines. J Cell Physiol 193: 244-252, 2002.

23. Ciampolillo A, De Tullio C and Giorgino F: The IGF-I/IGF-I receptor pathway: Implications in the pathophysiology of thyroid cancer. Curr Med Chem 12: 2881-2891, 2005.

24. Surmacz E and Bartucci M: Role of estrogen receptor alpha in modulating IGF-I receptor signaling and function in breast cancer. J Exp Clin Cancer Res 23: 385-394, 2004.

25. Garcia-Solis P and Aceves C: 5'Deiodinase in two breast cancer cell lines: Effect of triiodothyronine, isoproterenol and retinoids. Mol Cell Endocrinol 201: 25-31, 2003.

26. Dhiman VK, Bolt MJ and White KP: Nuclear receptors in cancer-uncovering new and evolving roles through genomic analysis. Nat Rev Genet 19: 160-174, 2018.

27. Kim WG and Cheng SY: Thyroid hormone receptors and cancer. Biochim Biophys Acta 1830: 3928-3936, 2013. 
28. Nugroho $\mathbf{J}$ and Schweiger B: Isotretinoin as a possible environmental trigger to autoimmunity in genetically susceptible patients. Case Rep Pediatr 2017: 4207656, 2017.

29. Schmutzler C, Brtko J, Winzer R, Jakobs TC, Meissner-Weigl J, Simon D, Goretzki PE and Köhrle J: Functional retinoid and thyroid hormone receptors in human thyroid-carcinoma cell lines and tissues. Int J Cancer 76: 368-376, 1998.

30. Hoftijzer HC, Liu YY, Morreau H, van Wezel T, Pereira AM, Corssmit EP, Romijn JA and Smit JW: Retinoic acid receptor and retinoid X receptor subtype expression for the differential diagnosis of thyroid neoplasms. Eur J Endocrinol 160: 631-638, 2009.

31. Haugen BR, Larson LL, Pugazhenthi U, Hays WR, Klopper JP, Kramer CA and Sharma V: Retinoic acid and retinoid X receptors are differentially expressed in thyroid cancer and thyroid carcinoma cell lines and predict response to treatment with retinoids. J Clin Endocrinol Metab 89: 272-280, 2004.
32. Kim D: The role of vitamin D in thyroid diseases. Int J Mol Sci 18: pii: E1949, 2017

33. Casula S and Bianco AC: Thyroid hormone deiodinases and cancer. Front Endocrinol (Lausanne) 3: 74, 2012.

34. Chen W, Wang S, Tian T, Bai J, Hu Z, Xu Y, Dong J, Chen F, Wang $X$ and Shen $\mathrm{H}$ : Phenotypes and genotypes of insulin-like grow th factor 1, IGF-binding protein-3 and cancer risk: Evidence from 96 studies. Eur J Hum Genet 17: 1668-1675, 2009.

35. Lin K, He S, He L, Chen J, Cheng X, Zhang G and Zhu B: Complement component 3 is a prognostic factor of non-small cell lung cancer. Mol Med Rep 10: 811-817, 2014. 